\title{
Drug-Based Disruption of Protein Complexes with Unknown Structure: Towards a Novel Platform for Drug Discovery
}

Ariel Fernández Stigliano $0^{1,2,3^{*}}$

${ }^{1}$ Argentine Institute of Mathematics, National Research Council (CONICET), Buenos Aires 1083, Argentina

${ }^{2}$ Collegium Basilea, Institute for Advanced Study, Basel 4053, Switzerland

3 Institute for Biophysical Dynamics, the University of Chicago, Chicago, IL 60637, USA

*Corresponding author: Ariel Fernández Stigliano, Institute for Biophysical Dynamics, The University of Chicago, Chicago, IL 60637, USA, Tel: 54 11 49546781; Fax: 54114954 6782; E-mail: ariel@afinnovation.com

Received date: September 29, 2015; Accepted date: September 30, 2015; Published date: October 04, 2015

Copyright: $\odot 2015$ Stigliano AF. This is an open-access article distributed under the terms of the Creative Commons Attribution License, which permits unrestricted use, distribution, and reproduction in any medium, provided the original author and source are credited.

\section{Editorial}

The recruitment of a protein complex constitutes the most elementary molecular event in the biology of the cell [1]. However, the disruption of such complexes with small-molecule ligands poses a major problem in drug discovery. The problem becomes even more difficult when the structure of the target protein is unknown. Based solely on protein sequence, a new discovery platform is emerging to identify drug leads to disrupt protein-protein associations (PPAs). Here we review this technology as applied in a recent invention.

When PPAs involve binding partners altered in a disease-related context, complex formation may lead to deregulation of biological function and a drug-based disruption of the complex represents a therapeutic opportunity [2-4]. However, major problems arise in the identification of drug leads and optimization strategies for small PPAdisruptive compounds [4]. The PPA regions often extend over more than $1000 \AA 2$ on the protein surface; in contrast with the smaller binding sites for natural ligands bind [5]. Thus, the absence of obvious leads like natural ligands and the size of the binding surface make it difficult to identify candidate compounds that may be optimized into therapeutic agents to disrupt PPAs.

To address the problems related to epitope size in PPAs, methodologies have been implemented for the identification of "hot spots" that make the most significant contribution to binding [6]. Such approaches are usually based on alanine scanning, assessing the impact of individual residues on the binding free energy. In this way, an effective epitope significantly smaller than the PPA region is determined [4,7] and fragment-based lead discovery may be subsequently exploited to generate candidate ligands for competitive binding $[4,8,9]$.

The problem of therapeutic disruption of a PPA becomes especially difficult when the structure of the targeted binding partner is unknown. As argued in this editorial piece, even in such cases, it is possible to implement a drug discovery platform based solely on sequence-based predictors of binding epitopes. The novel drugdiscovery platform hinges on two premises: a) structural defects in proteins, known as dehydrons, promote water exclusion at the interface, and thus residues paired by dehydrons constitute PPA hot spots [10]; b) dehydrons are identified as order-disorder twilight regions along the protein sequence [11] and therefore may be inferred utilizing a sequence-based predictor of intrinsic disorder [12]. The efficacy of the technology will be illustrated by a recently patented invention to treat heart failure through disruption of the myosinMyBP-C interface [13]. MyBP-C refers to a multi-domain myosin- binding protein with unreported structure that is a central regulator of cardiac contractility. MyBP-C molecules constitute molecular brakes modulating the displacement of myosin motors. By sequence-based inference of dehydron-rich regions in MyBP-C, we identified drug leads to cure heart failure. Here we describe the technological advances utilized in the invention to enable therapeutic disruption of PPAs.

In regards to the scientific foundations of the novel technology, we should first point out that the integrity of a soluble protein is contingent on the capability of its structure to exclude water from backbone amide-carbonyl hydrogen bonds. Water-exposed intramolecular hydrogen bonds, the so-called dehydrons, constitute structural defects taking the particular form of wrapping deficiencies [11]. By wrapping we mean the extent to which the backbone hydrogen bond is shielded from hydration. Dehydrons favor removal of surrounding water to strengthen and stabilize the underlying electrostatic interaction, and thus are predictably implicated in PPAs. By exogenously contributing to the wrapping of pre-formed hydrogen bonds, PPAs in effect remove pre-existing dehydrons, a picture supported by bioinformatics evidence [10]. Thus, dehydrons have been identified as decisive factors driving complex formation and proteins with dehydron-rich regions must rely on binding partnerships to maintain their structural integrity. On the other hand, dehydron-rich regions identified on the protein sequence may be characterized as belonging to the "twilight zone" between order and native disorder [11]. This characterization is suggested by a strong correlation between wrapping of intramolecular hydrogen bonds and propensity for structural disorder. The correlation reflects the fact that a local inability to exclude water intramolecularly from pre-formed hydrogen bonds is causative of a local loss of structural integrity, whereby full backbone hydration becomes structurally disruptive.

The local disorder propensity may be quantified by a sequencebased score generated by a predictor of native disorder propensity [12]. The disorder score $(0 \leq \mathrm{fd} \leq 1)$ is assigned to each residue within a sliding window, representing the predicted propensity of the residue to be in a disordered region ( $\mathrm{fd}=1$, certainty of disorder; $\mathrm{fd}=0$, certainty of order). A strong correlation between disorder score of a residue and extent of wrapping of the hydrogen bond engaging the residue implies that dehydrons correspond to structurally vulnerable regions [11]. Thus, a characterization of dehydrons as belonging to the orderdisorder twilight range $0.35<\mathrm{fD}<0.8$ and flanked by ordered and disordered regions is warranted.

The picture put forth in the preceding paragraphs makes it intuitively appealing to identify dehydron-rich regions with peptidebased leads to disrupt PPAs [13]. To illustrate the power of the 
Citation: Stigliano AF (2015) Drug-Based Disruption of Protein Complexes with Unknown Structure: Towards a Novel Platform for Drug Discovery. J Pharmacogenomics Pharmacoproteomics 6: e149. doi:10.4172/2153-0645.1000e149

Page 2 of 2

discovery technology, we focus on the lead identification for disruption of a specific PPA, a patented invention to treat heart failure [13].

Myosin binding protein $\mathrm{C}$ (MyBP-C) is a central regulator of cardiac function [13]. In animal models, genetic ablation or phosphorylation of MyBP-C accelerates contraction and increases its force in cardiac muscle. Most recently, it has been shown that phosphorylation of MyBP-C at Ser282 and Ser302 in the mouse or Ser284 and Ser304 in the human underlies the increase in myocardial contractile force as heart rate is increased. MyBP-C normally reduces the speed and strength of contraction by means of its interaction with the protein motor myosin, thereby reducing the probability of myosin sliding along actin filaments. Ablation or phosphorylation of MyBP-C disrupts this interaction and relieves MyBP-C repression of myosin. Once phosphorylated, MyBP-C no longer binds to myosin, myosin slides along actin, and the contraction speed and strength increase.

In heart failure, MyBP-C is phosphorylated minimally or not at all. Since phosphorylation of MyBP-C improves contraction, the inventors in US patent 9,051,387 [13] proposed to target the phosphorylation site on MyBP-C with a pharmaceutical designed to disrupt its repressive interaction with myosin. This problem involves designing an optimal MyBP-C-derived peptide. The peptide contains the motif responsible for interaction with myosin and hence disrupts the MyBP-C-myosin repressive association.

The 3D-structure of the multi-domain protein MyBP-C is unknown, and therefore it was decided to design the peptide based on the output from $\mathrm{PONDR}^{\oplus}$, a predictor of native disorder $[12,13]$. The peptide was developed based on a sequence-based prediction of the dehydron-rich region in $\mathrm{MyBP}-\mathrm{C}$ that purportedly constitutes the putative myosinbinding site. To predict the peptide sequence, the inventors examined a region between the $\mathrm{C} 1$ and $\mathrm{C} 2$ domains of MyBP-C that lies in the twilight zone between order and disorder. In this way, it became possible to identify the dehydron-rich region containing modulating phosphorylation sites in the motif region intercalated between domains $\mathrm{C} 1$ and $\mathrm{C} 2$ of $\mathrm{cMyBP}-\mathrm{C}$ and determine the sequence of the (patented) peptide that may be used as a lead for the drug-based treatment of heart failure.

We have reported on new developments in the sequence-based prediction of protein regions that generate water-protein interfacial tension. Such advances usher the advent of a novel drug-discovery platform while supporting the view that it is possible to identify therapeutic leads to disrupt the recruitment of protein complexes in the absence of 3D-structure.

This research was made possible through financial support from AF Innovation, a Pharmaceutical Consultancy.

\section{References}

1. Pelay-Gimeno M, Glas A, Koch O, Grossmann TN (2015) Structure-Based Design of Inhibitors of Protein-Protein Interactions: Mimicking Peptide Binding Epitopes. Angew Chem Int Ed Engl 54: 8896-8927.

2. Wells JA, McClendon CL (2007) Reaching for high-hanging fruit in drug discovery at protein-protein interfaces. Nature 450: 1001-1009.

3. Nero TL, Morton CJ, Holien JK, Wielens J, Parker MW (2014) Onco-genic protein interfaces: small molecules, big challenges. Nature Rev Cancer 14: 248-262.

4. Arkin MR, Tang Y, Wells JA (2014) Small-molecule inhibitors of proteinprotein interactions: Progressing toward the reality. Chem Biol 21: 1102-1114.

5. Hwang H, Vreven T, Janin J, Weng Z (2010) Protein-protein docking benchmark version 4.0. Proteins 78: 3111-3114.

6. Kouadio JL, Horn JR, Pal G, Kossiakoff AA (2005) Shotgun Alanine Scanning Shows That Growth Hormone Can Bind Productively to Its Receptor through a Drastically Minimized Interface. J Biol Chem 280: 25524-25532.

7. King C, Garza EN, Mazor R, Linehan JL, Pastan I, et al. (2014) Removing T-cell epitopes with computational protein design. Proc Natl Acad Sci USA 111: 8577-8582.

8. Erlanson DA, Wells JA, Braisted AC (2004) Tethering fragment-based drug discovery. Ann Rev Biophys Biomol Struct 33: 199-223.

9. Winter A, Higueruelo AP, Marsh M, Sigurdardottir A, Pitt WR, et al. (2012) Biophysical and computational fragment-based approaches to targeting protein-protein interactions: applications in structure-guided drug discovery. Q Rev Biophys 45: 383-426.

10. Fernández A (2012) Epistructural tension promotes protein associations. Phys Rev Lett 108: 188102.

11. Fernández SA (2015) Proteins in the order-disorder twilight: Unstable interfaces promote protein aggregation. Biomolecular Interfaces 97-124.

12. Xue B, Dunbrack RL, Williams RW, Dunker AK, Uversky VN (2010) PONDR-FIT: a meta-predictor of intrinsically disordered amino acids. Bioch Biophys Acta 1804: 996-1010.

13. Moss R, Fernández A (2015) Inhibition of MyBP-C binding to myosin as a treatment for heart failure. US patent. 